\title{
In-hospital cardiac arrest in hospitals with mature rapid response systems - a multicentre, retrospective cohort study
}

\section{Tirkkonen, Joonas}

2020-04

Tirkkonen , J , Skrifvars , M B , Parr , M , Tamminen , T \& Aneman , A 2020 , ' In-hospital cardiac arrest in hospitals with mature rapid response systems - a multicentre, retrospective cohort study ' , Resuscitation, vol. 149 , pp. 109-116 . https://doi.org/10.1016/j.resuscitation.2020.02.022

http://hdl.handle.net/10138/327188

https://doi.org/10.1016/j.resuscitation.2020.02.022

cc_by_nc_nd

acceptedVersion

Downloaded from Helda, University of Helsinki institutional repository.

This is an electronic reprint of the original article.

This reprint may differ from the original in pagination and typographic detail.

Please cite the original version. 


\section{In-hospital cardiac arrest in hospitals with mature rapid response systems - a multicentre, retrospective cohort study}

Joonas Tirkkonen, MD, PhD. Department of Intensive Care Medicine and Department of Emergency, Anaesthesia and Pain Medicine, Tampere University Hospital, Tampere, Finland and Intensive Care Unit, Liverpool Hospital, Sydney, Australia. PO Box 2000, FI-33521 Tampere, Finland. joonas.tirkkonen@tuni.fi

Markus B Skrifvars, MD, PhD, Professor. Department of Emergency Care and Services, University of Helsinki and Helsinki University Hospital, Helsinki, Finland. PO Box 22, FI-00014 Helsinki, Finland. markus.skrifvars@hus.fi

Michael M Parr, MD, Conjoint Professor. Intensive Care Unit, Liverpool Hospital, Sydney, Australia and University of New South Wales, South Western Sydney Clinical School, Sydney, Australia. Elizabeth Street, Liverpool, NSW 2170, Australia. michael.parr@health.nsw.gov.au

Tero Tamminen, MD. Division of Intensive Care, Department of Anaesthesiology, Intensive Care and Pain Medicine, Helsinki University and Helsinki University Hospital. PO Box 22, FI-00014 Helsinki, Finland. tero.tamminen@ @us.fi

Anders Aneman, MD, PhD, Conjoint Professor. Intensive Care Unit, Liverpool Hospital, Sydney, Australia; University of New South Wales, South Western Sydney Clinical School, Sydney, Australia; Macquarie University, Faculty of Medicine and Health Sciences, Sydney, Australia. Elizabeth Street, Liverpool, NSW 2170, Australia. anders.aneman@ health.nsw.gov.au

\section{Corresponding author:}

Joonas Tirkkonen, MD, PhD. Department of Intensive Care Medicine and Department of Emergency, Anaesthesia and Pain Medicine, Tampere University Hospital, Tampere, Finland and Intensive Care Unit, Liverpool Hospital, Sydney, Australia. PO Box 2000, FI-33521 Tampere, Finland.

joonas.tirkkonen@tuni.fi $+3583311611$

Word count abstract: 247

Word count article: 2,747

Tables and figures: 6

Appendices: 2

Number of references: 26

Key words: in-hospital cardiac arrest; rapid response team; rapid response system 


\begin{abstract}
Aim: To investigate in-hospital cardiac arrests (IHCAs) according to the Ustein template in hospitals with mature systems utilizing rapid response teams (RRTs), with a special reference to preceding RRT factors and factors associated with a favourable neurological outcome (cerebral performance category (CPC) 1-2) at hospital discharge.
\end{abstract}

Methods: Multicentre, retrospective cohort study between 2017-2018 including two Finnish and one Australian university affiliated tertiary hospitals.

Results: A total 309 IHCAs occurred with an incidence of 0.78 arrests per 1,000 hospital admissions. The median age of the patients was 72 years, $63 \%$ were male and $73 \%$ had previously lived a fully independent life with a median Charlson comorbidity index of two. Before the IHCA, $16 \%$ of the patients had been reviewed by RRTs and $26 \%$ of the patients fulfilled RRT activation criteria in the preceding 8 hours of the IHCA. Return of spontaneous circulation was achieved in $53 \%$ of the patients and $28 \%$ were discharged from hospital with CPC 1-2. In a multivariable model, younger age, no pre-arrest RRT criteria, arrest in normal work hours, witnessed arrest and shockable initial rhythm were independently associated with CPC 1-2 at hospital discharge.

Conclusions: In hospitals with mature rapid response systems most IHCA patients live a fully independent life with low burden of comorbid diseases before their hospital admission, the IHCA incidence is low and outcome better than traditionally believed. Deterioration before IHCA is present in a significant number of patients and improved monitoring and earlier interventions may further improve outcomes. 


\section{INTRODUCTION}

Studies of in-hospital cardiac arrest (IHCA) have been infrequent in resuscitation science. ${ }^{1,2}$ Most IHCA studies report results from cohorts over a decade old, and reports of patient-centered outcomes beyond survival, e.g. including favourable neurological outcome, are rare. ${ }^{1,2}$ Some more recent multicentre registry studies have demonstrated the status quo of European in-hospital resuscitation with IHCA incidence of 1.5-1.8 arrests per 1,000 hospital admissions and 15-18\% of IHCA patients surviving to hospital discharge. ${ }^{3-5}$ However, detailed data on pre-, peri- and postarrest factors as well as neurological outcomes are still lacking. The International Liaison Committee on Resuscitation (ILCOR) published new Utstein Guidelines for IHCA on November 2019 underlining the precise recording of core and supplemental data elements. ${ }^{6}$

While the acuity and morbidity of hospitalized patients have increased substantially as a result of expanding treatment possibilities in recent decades, the incidence of IHCA seems to have remained stable or even declined. ${ }^{3,7}$ The international adaptation of rapid response systems (RRSs) into hospitals' standard operating procedures may in part explain this trend. ${ }^{8}$ Many IHCAs today occur RRSs, but only one previous study has investigated the interface between RRS factors and subsequent IHCAs by cross-linking hospital registries in the United States in 2000-2014 suggesting missed intervention opportunities in many RRS hospitals. ${ }^{9}$

In this multicentre study from two Finnish and one Australian university-affiliated tertiary hospitals with mature RRSs we aimed 1) to describe in detail the core and supplemental data of in-hospital cardiac arrest as suggested in ILCOR statement, ${ }^{6}$ 2) to document RRS factors preceding the cardiac arrests and 3) to investigate the factors independently associated with a favourable neurological outcome at hospital discharge. 


\section{METHODS}

\subsection{Ethics}

The Ethics Committees of the Tampere University Hospital (TAYS) (Approval no: R18203), Helsinki University Hospital (HUS) (HUS/1493/2019) and South Western Sydney Local Health District (LNR/19/LPOOL/11) all approved the study protocol.

\subsection{Hospitals}

TAYS and HUS Meilahti are two of the five university affiliated tertiary referral centers in Finland, providing the most advanced care for a catchment population of three million citizens. Liverpool Hospital is a university affiliated principal referral hospital providing tertiary care for the greater South Western Sydney residents. These hospitals together provide 1,700 beds and receive 197,000 annual admissions; detailed hospital characteristics are presented in Appendix A.

\subsection{Rapid response systems}

Liverpool hospital introduced the Medical Emergency Team (= the first rapid response team, RRT) in 1990 and has over 25 years of operational experience, while TAYS and HUS Meilahti were the first Finnish tertiary hospitals to implement RRTs ten years ago (Appendix A). The RRTs at these hospitals are available to attend IHCAs as well as other medical emergencies for twenty-four hours seven days a week. All hospitals utilized quite similar track and trigger RRT criteria for team activation in 2017-2018 with minor differences in the upper threshold for respiratory rate $(24,28$ and 30 breaths per minute, respectively, Appendix A). In addition, in 2010 Liverpool hospital implemented the 'between the flags' criteria to enable earlier detection of deviations in vital signs triggering ward level consultation. ${ }^{10}$ If a patient fulfills the 'between the flags' criteria (Appendix A), a 'clinical review call' should be made alerting ward's medical officer. TAYS, on the other hand, begun implementing the national early warning score for patient monitoring in $2017 .{ }^{11}$ 
No standard definition for a 'mature RRS' exist, but all three hospitals have educated and trained ward staff for more than 10 years to monitor vital signs and recognize any compromise; have used governance systems for the RRS since implementation; and have established academic programs to evaluate the RRS.

\subsection{Definitions}

IHCA refers to cessation of cardiac activity requiring chest compressions and/or defibrillation in a hospitalized patient who had a pulse at the time of hospital admission. Activities of daily living (ADLs) refers to the basic daily routines required for normal self-care (eating, personal hygiene, continence, getting dressed, moving around in the house etc). The Charlson comorbidity index (CCI) was used to define patients' cumulative comorbidity. ${ }^{12}$ The cerebral performance category (CPC) was applied to describe the neurological state of patients both at hospital admission and at hospital discharge. ${ }^{13}$ The neurological outcome was dichotomized into CPC 1-2 (at worst moderate neurological disability but independent in basic activities of daily living) versus CPC 3-5 (composite of poor neurological function or death). 'The variable 'RRT criteria 0 - $8 \mathrm{~h}$ before the arrest' means that a patient has fulfilled hospitals' RRT activation criteria during the eight hours preceding the IHCA but RRT has not been activated due to these observed abnormal vital signs. This has been referred as 'afferent limb failure' or 'missed RRT opportunity' in the previous literature. $^{9}$

\subsection{Exclusion criteria}

Patients were excluded where they were: under 18 years of age, arrived in cardiac arrest to the hospital, or arrested in ICU. Only the first arrest call was included for patients with multiple resuscitation events.

\subsection{Data collection}


All three hospitals collect prospectively data on RRT activations for governance and research purposes. This study investigated RRT calls between $1^{\text {st }}$ of January 2017 to $31^{\text {st }}$ of December 2018 and included all activations for IHCAs not meeting the exclusion criteria (above). While the periarrest factors were prospectively recorded on site to an RRT template and inputted to the ICUs' electronic patient management systems, the detailed data on pre-arrest factors and hospital outcomes were retrospectively retrieved from the electronic patient notes.

\subsection{Statistical analyses}

Data are presented as percentages with counts or as medians with interquartile ranges. First, factors previously reported to be associated with IHCA outcome, ${ }^{2-5,7,9}$ as well as all factors judged clinically relevant by the study group, were presented to a univariate model. Univariate logistic regression was used to identify factors plausibly associated with good neurological outcome at hospital discharge with an initial $p$-value of $<0.20$. All factors with $p<0.20$ were checked to verify an event rate $\geq 5$ per candidate variable ${ }^{14}$ and introduced to a multivariable logistic regression model with forward likelihood ratio method. The goodness-of-fit of the multivariable model was tested with the Hosmer-Lemeshow Chi-Square analysis and odds ratios with two-sided p-values $<0.05$ and with $95 \%$ confidence intervals not including the value 1.00 were considered to represent statistically significant results. SPSS version 25 for Windows (SPSS Inc., Chicago, IL, USA) was used for all statistical analyses. 


\section{RESULTS}

\subsection{Cohort description}

A total of 8,023 adult RRT activations occurred during the study period including 309 IHCAs (Figure 1). The annual incidence of IHCA was 0.78 per 1,000 hospital admissions while the RRT activation rate per 1,000 hospital admissions was 20 (for individual hospitals please see Appendix A).

Most IHCA patients were male, had a median age of 72 years, a median CCI of two and $73 \%$ lived a fully independent life before admission to hospital (Table 1). Patients had been admitted a median three days before the IHCA. Sixteen percent of the patients had had a preceding RRT call during their hospital admission with $4.5 \%$ occurring in the preceding 24 hours. One in four patients (26\%) had met RRT activation criteria but had no RRT review in the eight hours preceding the arrest. Appendix B presents the patient data for individual hospitals.

\subsection{Cardiac arrest characteristics}

The peri-arrest characteristics are provided in Table 2 . While $87 \%$ of the patients were either in arrest or had already been resuscitated at RRT arrival, 14\% progressed to IHCA after the arrival of the RRT. Three quarters of the IHCAs were witnessed, $39 \%$ had an identifiable cardiac cause and $16 \%$ had an initial cardiac rhythm that was shockable. A mechanical chest compression device (Lucas®) was applied in $6.5 \%$ of patients.

\subsection{Patient outcomes}

Return of spontaneous circulation (ROSC) was achieved in 53\% of IHCAs and $68 \%$ of patients with ROSC were transferred to ICU with a median stay of three days (Table 3). Eighty-nine patients survived to hospital discharge out of whom $96 \%$ had CPC1-2. Figure 2 summarizes the RRTassociated characteristics and clinical trajectories for the 309 IHCAs. 


\subsection{Factors independently associated with favourable neurological outcome at hospital}

\section{discharge}

Eleven clinically plausible variables with $p<0.20$ on univariate testing were included in the multivariable regression model for favourable neurological outcome, CPC 1-2, at hospital discharge (Table 4). Increasing age, presence of RRT activation criteria $0-8 \mathrm{~h}$ before the IHCA without RRT activation and arrest during on-call hours decreased the odds for survival with CPC 1-2 whereas witnessed arrest and shockable initial rhythm increased the odds for being discharged with CPC 12. 


\section{DISCUSSION}

\subsection{Key findings}

In this observational, multicenter cohort study of IHCA in hospitals with well-established RRSs we found a low IHCA incidence with patients demonstrating relatively few comorbidities and the majority living a fully independent life before admission to hospital. One in six IHCA patients had been reviewed previously by the RRT, but one in four had met RRT activation criteria without subsequent RRT activation in the preceding eight hours. About a quarter of IHCA patients were discharged with a favourable neurological outcome. Unwitnessed arrest and preceding RRT criteria without RRT activation were independently associated with poor neurological state or death and remain significant contributors to poor outcomes despite the operation of mature RRSs.

\subsection{Incidence}

Recent registry studies suggest that the incidence of IHCAs is between 1.5-1.8 per 1,000 hospital admissions in Europe, while in the United States the incidence is currently substantially higher with 9-10 IHCAs per 1,000 admissions. ${ }^{2-5}$ We observed the IHCA incidence to be less than half of that reported in European registries. ${ }^{3-5}$ These registry studies, however, include ICU cardiac arrests (ICUCAs), which represent $7-16 \%$ of the cases, but were excluded in this study and hence the higher incidence rates are to some extent expected. ${ }^{3-5}$ In the context of RRSs that aim to facilitate ICU admission of deteriorating patients before progression to IHCA or indeed to implement treatment limitations to avoid unethical resuscitation attempts, it can be argued that ICUCAs should be reported separately from other IHCAs. ${ }^{15}$ The more relevant IHCA incidence rates for comparison therefore come from RRS studies reporting the incidence rates before and after the RRS implementation. In a meta-analysis by Chan et al. including 13 RRS studies published before November 2008, the non-ICU IHCA incidence rates after the RRS implementation varied between 1.5-5.3 per 1,000 hospital admissions. ${ }^{16}$ A more recent meta-analysis by Maharaj et al. including 
RRS studies up to December 2013 found the combined non-ICU IHCA incidence to be 2.1 per 1,000 hospital admissions after the RRS implementation, but also documented the fact that the IHCA incidence was recorded just 0-24 months after the RRS implementation in all studies. ${ }^{17}$ The lower incidence found in our current study might reflect that it takes years of continuous system development for the RRS achieve its full potential.

\subsection{Patient and peri-arrest characteristics}

Very limited data exist on the pre-arrest functional status of IHCA patients, whilst needed for a more complete understanding of this patient population. Chan et al. found that $18 \%$ of the IHCA patients in the United States had a pre-arrest CPC score of 3 or higher, which is strikingly higher compared with the $2 \%$ of patients reported in this study on admission to hospital. ${ }^{18} \mathrm{~A}$ recent Italian cohort study reported pre-arrest CPC scores $>2$ for eight percent of patients. ${ }^{4}$ These differences likely reflect sociocultural differences in treatment limitation policies. ${ }^{2}$

Most patients lived fully independently prior to hospital admission. This is an important descriptive factor for IHCA patients as it substantially influences the perceived quality of life. ${ }^{19}$ One previous study reported the preceding functional status of IHCA patients, but only in survivors with good neurology ${ }^{20}$ which precludes meaningful comparisons with this study. Furthermore, the cumulative comorbidity of IHCA patients is often not reported. Our finding of a relatively low cumulative comorbidity was identical to that observed in a large Danish registry study. ${ }^{5}$

Pre-arrest abnormal vital signs decrease the chances of survival from IHCA, and in hospitals with mature RRSs preceding deterioration that has not resulted in RRT review should be rare. ${ }^{21}$ The presence of RRT criteria without timely RRT calls, referred to as afferent limb failure, is dependent both on the RRT criteria per se and the maturity of the RRS. ${ }^{22}$ The argument of maturity notwithstanding, one in four IHCA patients in this study had recorded RRT review criteria prearrest without sufficient action. Chan et al. reported that $17 \%$ of IHCA patients were reviewed by 
the RRT within 24 hours before the arrest, ${ }^{9}$ which is about four times more frequent as compared with the number of IHCA patients reviewed in the preceding 24 hours in this study. In one in ten patients, the RRT call was made for abnormal vital signs rather than IHCA while a similar proportion of patients finally progressed to arrest after arrival of the RRT. Thus there were clearly instances of afferent limb failure in this study and this aspect is important for future IHCA studies that should report preceding vital signs abnormalities, preceding RRT activations as well as the RRT calling reasons for a better understanding on the 'modern' in-hospital cardiac arrest in RRS hospitals.

\subsection{Patient outcomes and factors associated with good neurological survival}

The IHCA survival to hospital discharge has been reported as between $15-18 \%$ in Italy and the United Kingdom and 22-23\% in the United States, ${ }^{3,4,23}$ with $85-91 \%$ of hospital survivors in CPC 12 at discharge when reported. ${ }^{2,4}$ A recent Danish registry documented as high as $28 \%$ 30-day survival, although no data on neurological recovery was available. According to most recent data the overall IHCA survival has improved and our results are in line with this trend with $28 \%$ of the IHCA patients surviving to discharge with good neurology. It is also encouraging that only four out of 309 IHCA patients were discharged with poor neurological outcome. Keeping in mind the obvious ethical and mental burden that survival with a CPC 3-4 causes to patients' relatives and the community, a recent study reported a 1.7-fold increase in one-year healthcare costs for IHCA patients with CPC 3-4 as compared with IHCA patients with CPC 1-2. ${ }^{24}$

Several factors associated with survival from IHCA are either well established (e.g. shockable initial rhythm) or not amenable to intervention (e.g. age, comorbidities, reason for hospital admission). ${ }^{2,18}$ The multivariable model used in this study identified positive RRT criteria within eight hours of IHCA without subsequent RRT activation and unwitnessed arrest to be independently associated with decreased chances for survival with favourable neurology. A recent systematic review found that in poorly-functioning RRSs the operational limbs (RRTs, ward staff) are left in 
environments with tenuous RRS training and unclear protocols and capabilities for patient monitoring and escalation of treatment. ${ }^{25}$ It may be debatable if it is possible to totally avoid IHCAs by just optimising the rapid response system factors, as for example, both the present data and previous studies show that the incidence of 'afferent limb failure' will persist even in mature RRSs. The IHCAs with unknown no-flow times (the unwitnessed IHCAs) have the poorest odds for survival while still representing one quarter of general ward arrests even in modern university affiliated tertiary centres. This clearly puts continuous patient monitoring in focus including the potential for compact wireless patient monitoring on general wards. ${ }^{26}$

\subsection{Study limitations}

This study has important strengths and limitations. It was conducted in three large university affiliated tertiary hospitals in two countries with different healthcare contexts and data were collected using the recently updated Utstein template for IHCA. ${ }^{6}$ Both factors enhance the external validity of our results. The study is limited by its observational design allowing for associations to be discussed but without any inference of causality. Internal validity is further limited due to minor, but important differences in hospitals policies in responding to patient deterioration. While decadelong experience of RRS were in place in all hospitals, the determination of what constitutes a mature RRS is arbitrary, although in this study it is supported by the hospitals' RRS characteristics and low IHCA incidences. The frequency of do-not-attempt-resuscitation orders was not captured while inevitably affecting IHCA rates. Finally, while CPC at hospital discharge is a patient centered outcome measure, the most important outcome for patients is arguably their preserved independence in the activities of daily living that we were unable to ascertain after hospital discharge.

\section{CONCLUSIONS}


The incidence of in-hospital cardiac arrest in hospitals with mature RRSs was low with comparatively better outcomes than reported in current IHCA literature. The IHCA patients had relatively low premorbid conditions and were often fully independent in ADLs before hospital admission. Future efforts aiming to further improve IHCA outcomes should underline the importance of timely RRT activation and focus on the possibilities of more continuous patient monitoring.

\section{Conflict of interest statement:}

All authors declare that no financial or non-financial conflicts of interests related to the submitted work exist.

\section{Acknowledgements}

JT has received research grants from Päivikki and Sakari Sohlberg Foundation and Instrumentarium Science Foundation. These Foundations had no role in the study design, in the collection, analysis and interpretation of data, in the writing of the manuscript or in the decision to submit the manuscript for publication. 


\section{REFERENCES}

1. Schluep M, Gravesteijn BY, Stolker RJ, Endeman H, Hoeks SE. One-year survival after inhospital cardiac arrest: A systematic review and meta-analysis. Resuscitation 2018;132:90100.

2. Andersen LW, Holmberg MJ, Berg KM, Donnino MW, Granfeldt A. In-Hospital Cardiac Arrest: A Review. JAMA 2019;321:1200-10.

3. Nolan J, Soar J, Smith GB, et al. Incidence and outcome of in-hospital cardiac arrest in the United Kingdom National Cardiac Arrest Audit. Resuscitation 2014;85:987-92.

4. Radeschi G, Mina A, Berta G, et al. Incidence and outcome of in-hospital cardiac arrest in Italy: a multicentre observational study in the Piedmont Region. Resuscitation 2017;119:48-55.

5. Andersen LW, Holmberg MJ, Løfgren B, Kirkegaard H, Granfeldt A. Adult in-hospital cardiac arrest in Denmark. Resuscitation 2019;140:31-6.

6. Nolan JP, Berg RA, Andersen LW, et al. Cardiac Arrest and Cardiopulmonary Resuscitation Outcome Reports: Update of the Utstein Resuscitation Registry Template for In-Hospital Cardiac Arrest: A Consensus Report From a Task Force of the International Liaison Committee on Resuscitation (American Heart Association, European Resuscitation Council, Australian and New Zealand Council on Resuscitation, Heart and Stroke Foundation of Canada, InterAmerican Heart Foundation, Resuscitation Council of Southern Africa, Resuscitation Council of Asia). Resuscitation 2019;144:166-77.

7. Doherty Z, Fletcher J, Fuzzard K, Kippen R, Knott C, O'Sullivan B. Short and long-term survival following an in-hospital cardiac arrest in a regional hospital cohort. Resuscitation $2019 ; 143: 134-41$.

8. Lyons PG, Edelson DP, Churpek MM. Rapid response systems. Resuscitation. 2018;128:191-7. 
9. Chan ML, Spertus JA, Tang F, Jayaram N, Chan PS. Missed opportunities in use of medical emergency teams prior to in-hospital cardiac arrest. Am Heart J 2016;177:87-95.

10. Aneman A, Frost SA, Parr MJ, Hillman KM. Characteristics and outcomes of patients admitted to ICU following activation of the medical emergency team: impact of introducing a two-tier response system. Crit Care Med 2015;43:765-73.

11. The Royal College of Physicians. National Early Warning Score (NEWS) 2: Standardising the assessment of acute-illness severity in the NHS. London: RCP; 2017;1-77.

12. Charlson ME, Pompei P, Ales KL, MacKenzie CR. A new method of classifying prognostic comorbidity in longitudinal studies: development and validation. J Chronic Dis. 1987;40:373-83.

13. Phelps R, Dumas F, Maynard C, Silver J, Rea T. Cerebral performance category and longterm prognosis following out-of-hospital cardiac arrest. Crit Care Med 2013;41:1252-7.

14. Vittinghoff E, McCulloch CE. Relaxing the rule of ten events per variable in logistic and Cox regression. Am J Epidemiol 2007;165:710-8.

15. Tirkkonen J, Efendijev I, Skrifvars MB. Cardiac arrest in the Intensive Care Unit. In: Vincent JL, editor. Annual Update in Intensive Care and Emergency Medicine 2019. $1^{\text {st }}$ ed. Springer International Publishing; 2019, p. 113-23.

16. Chan PS, Jain R, Nallmothu BK, Berg RA, Sasson C. Rapid response teams: a systematic review and meta-analysis. Arch Intern Med 2010;170:18-26.

17. Maharaj R, Raffaele I, Wendon J. Rapid response systems: a systematic review and metaanalysis. Crit Care 2015;19:254.

18. Chan PS, Spertus JA, Krumholz HM, et al. A validated prediction tool for initial survivors of in-hospital cardiac arrest. Arch Intern Med. 2012;172:947-53.

19. Lyu W, Wolinsky FD. The Onset of ADL Difficulties and Changes in Health-Related Quality of Life. Health Qual Life Outcomes. 2017;15:217. 
20. Hellevuo H, Sainio M, Huhtala H, Olkkola KT, Tenhunen J, Hoppu S. Good quality of life before cardiac arrest predicts good quality of life after resuscitation. Acta Anaesthesiol Scand 2018;62:515-21.

21. Andersen LW, Kim WY, Chase M, et al. The prevalence and significance of abnormal vital signs prior to in-hospital cardiac arrest. Resuscitation 2016;98:112-7.

22. Calzavacca P, Licari E, Tee A, et al. The impact of Rapid Response System on delayed emergency team activation patient characteristics and outcomes--a follow-up study. Resuscitation. 2010;81:31-5.

23. Go AS, Mozaffarian D, Roger VL, et al. Heart disease and stroke statistics - 2014 update: a report from the American Heart Association. Circulation 2014;129:e28-e292.

24. Efendijev I, Folger D, Raj R, et al. Outcomes and healthcare-associated costs one year after intensive care-treated cardiac arrest. Resuscitation 2018;131:128-34.

25. Olsen SL, Søreide E, Hillman K, Hansen BS. Succeeding with rapid response systems - a never-ending process: A systematic review of how health-care professionals perceive facilitators and barriers within the limbs of the RRS. Resuscitation 2019;144:75-90.

26. Weenk M, Koeneman M, van de Belt TH, Engelen LJLPG, van Goor H, Bredie SJH. Wireless and continuous monitoring of vital signs in patients at the general ward. Resuscitation 2019;136:47-53. 


\section{TITLES AND LEGENDS TO THE FIGURES}

\section{Figure 1.}

Title: Hospitals' RRT activations with the final cohort.

Legend: RRT, rapid response team; IHCA, in-hospital cardiac arrest.

\section{Figure 2.}

Title: IHCAs in the reference of preceding RRT factors and subsequent outcomes.

Legend: Read as a timeline from left to right: patients with preceding RRT calls during their hospitalization, RRT criteria without subsequent RRT activation before the IHCA, IHCA with ROSC or not, and the subsequent ICU admissions and hospital outcomes. RRT, rapid response team; IHCA, in-hospital cardiac arrest; ROSC, return of spontaneous circulation; ICU, intensive care unit; CPC, cerebral performance category. 
Table 1. Patient and hospital admission characteristics

Patient characteristics $\%$

Age (median; $\left.\mathrm{Q}_{1}, \mathrm{Q}_{3}\right)$

$72(60,81)$

Sex (male)

63

195

$\mathrm{CPC}<2$ before hospital admission

98

303

Performance in ADLs before hospital admission

Lives fully independently

Lives home with support in ADLs

23

Lives in nursing home, support in all ADLs

CCI (median; $\mathrm{Q}_{1}, \mathrm{Q}_{3}$ )

$2(1,4)$

Coronary artery disease

Peripheral arterial disease

Diabetes

Chronic obstructive pulmonary disease

11

Malignancy

18

54

Hospital admission characteristics

Inpatient admitted to hospital ward

95

294

Outpatient visiting a clinic

4.9

Surgical reason for admission*

Medical reason for admission

61

188

Days in hospital before the IHCA (median; $\mathrm{Q}_{1}, \mathrm{Q}_{3}$ )

$3(1,8.5)$

Preceding RRT call 16

Within 24 hours before the IHCA 


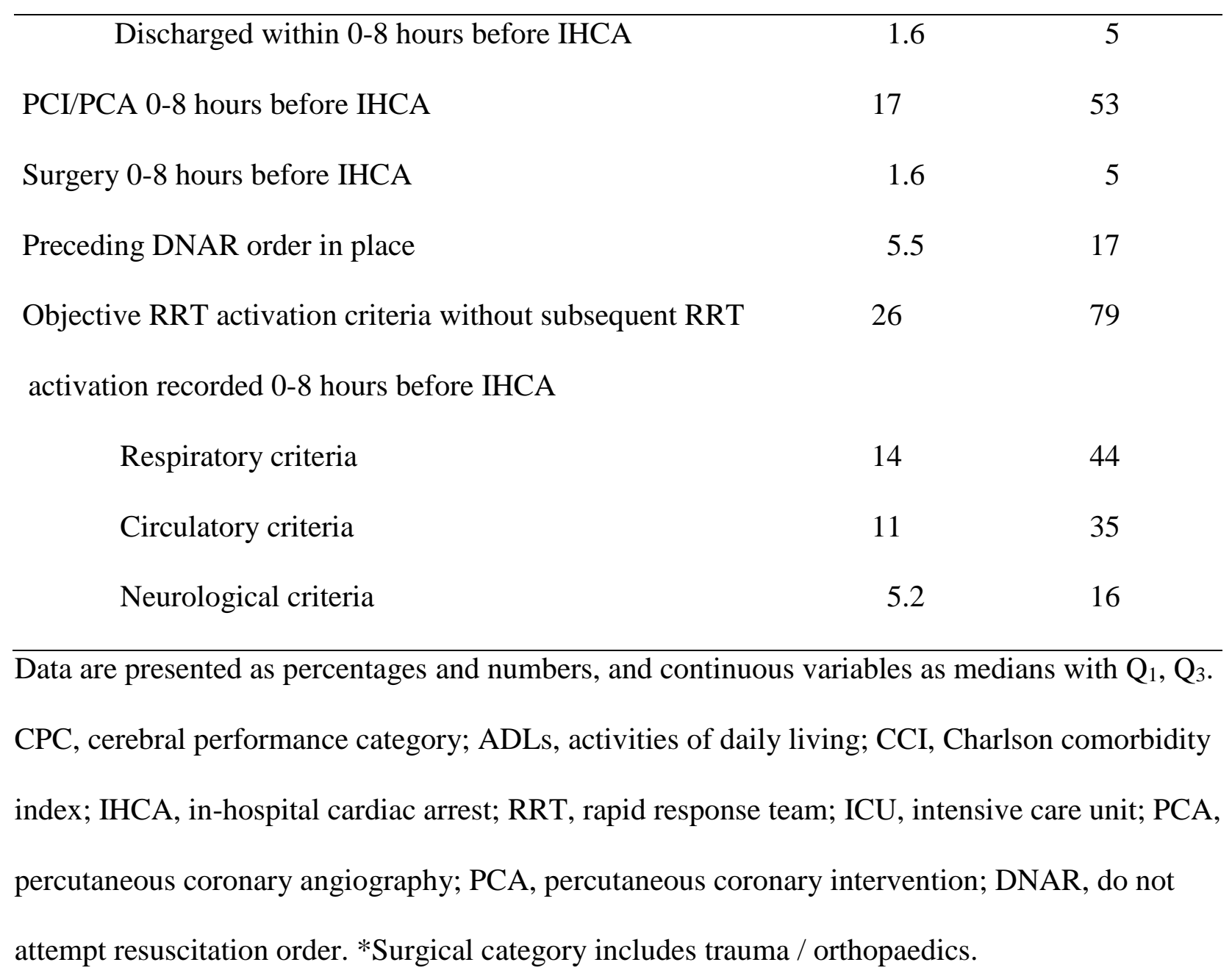


Table 2. Peri-arrest characteristics.

$\% \quad n$

RRT activation reason

Cardiac arrest

Respiratory criteria

4.2

13

Haemodynamic criteria

2.9

Neurological criteria

4.9

15

Nurse worried

0.3

1

Other

0.3

1

Location

Ward

83

256

Clinic

2.6

8

Angiology / Radiology

11

Emergency room

1.0

3

$\mathrm{OR} / \mathrm{PACU}$

2.3

7

RRT response time (min)

$2(2,3)$

Arrest during on-call hours

73

226

Situation at RRT arrival

Patient in cardiac arrest

76

Patient already with ROSC

11

Cardiac arrest after RRT arrival

14

42

Witnessed arrest

75

231

Reason for arrest

Cardiac

Respiratory

17 
Initial rhythm

Ventricular fibrillation

Ventricular tachycardia

Pulseless electrical activity

Asystole

Unknown

Duration of resuscitation attempt (min)

Defibrillation at any time during CPR

Adrenaline [epinephrine] at any time during CPR

Amiodarone at any time during CPR

Airway management

No airway management

Bag mask ventilation

Laryngeal mask

Endotracheal intubation

Tracheostomy already in place

Lucas® used

ECPR

Thoracostomy/ re-sternotomy conducted
12 38

$9.5(4,19)$

Data are presented as percentages and numbers, and continuous variables as medians with $\mathrm{Q}_{1}, \mathrm{Q}_{3}$. RRT, rapid response team; OR, operating room; PACU, post anaesthesia care unit; ROSC, return of spontaneous circulation; CPR, cardiopulmonary resuscitation; Lucas®, Lund University Cardiopulmonary Assist System; ECPR, extracorporeal cardiopulmonary resuscitation. 
Table 3. In-hospital cardiac arrest patient outcomes.

Patient outcome $\%$

ROSC

53

$n$

Post ROSC location

Same as arrest location

15

24

OR or PACU

4.3

7

High dependency unit

13

21

ICU

68

112

ICU length of stay

$3(1,6.5)$

ICU survival

58

66

24-hour survival

41

125

Survival to hospital discharge

29

89

Survival to hospital discharge with CPC 1-2

28

85

Data are presented as percentages and numbers, and continuous variables as medians with $\mathrm{Q}_{1}, \mathrm{Q}_{3}$.

ROSC, return of spontaneous circulation; OR, operating room; PACU, post anaesthesia care unit;

ICU, intensive care unit; CPC, cerebral performance category. 
Table 4. Univariate and multivariable logistic regression analyses of factors independently associated with a favorable neurological outcome (CPC 1-2) at hospital discharge.

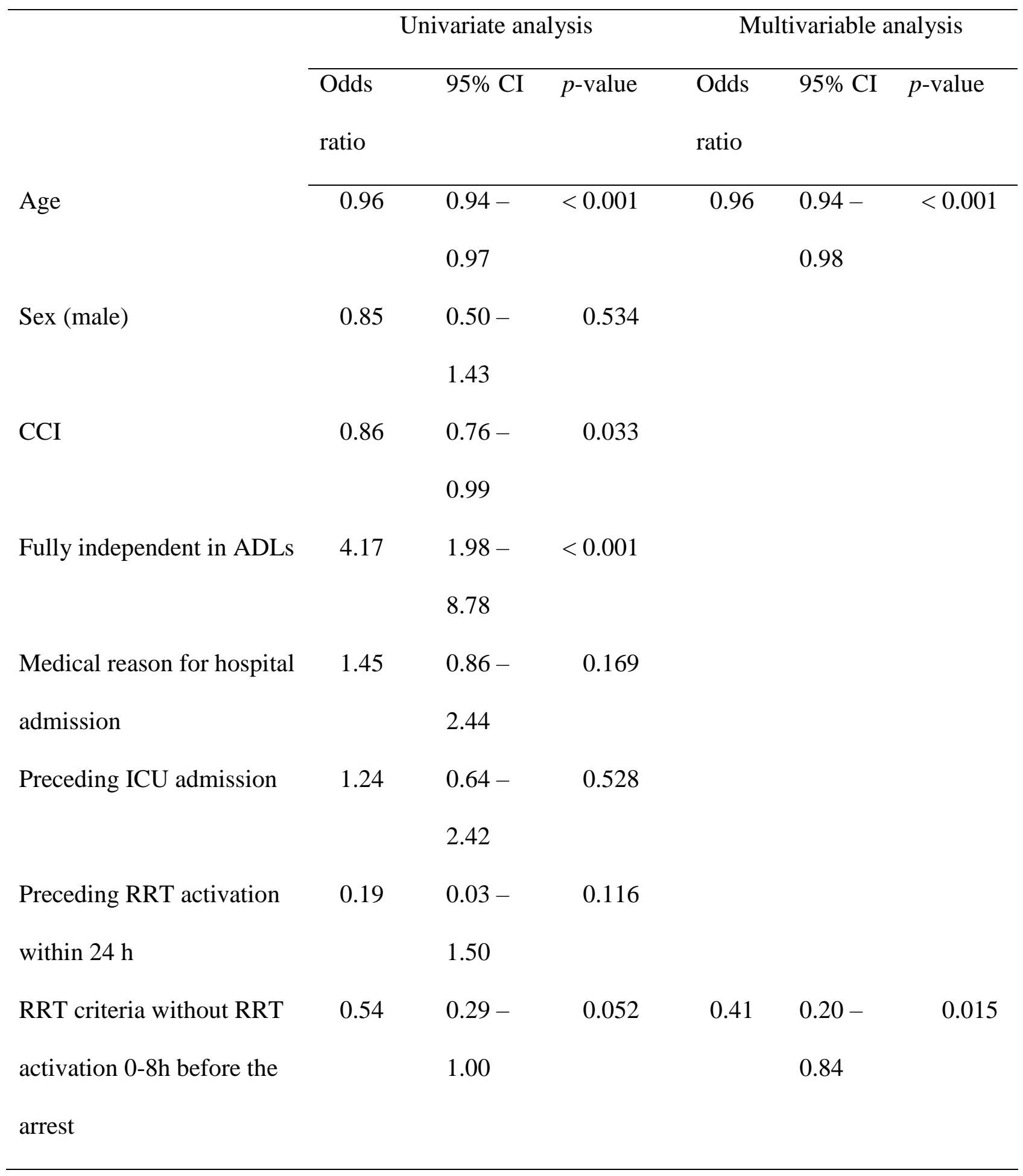




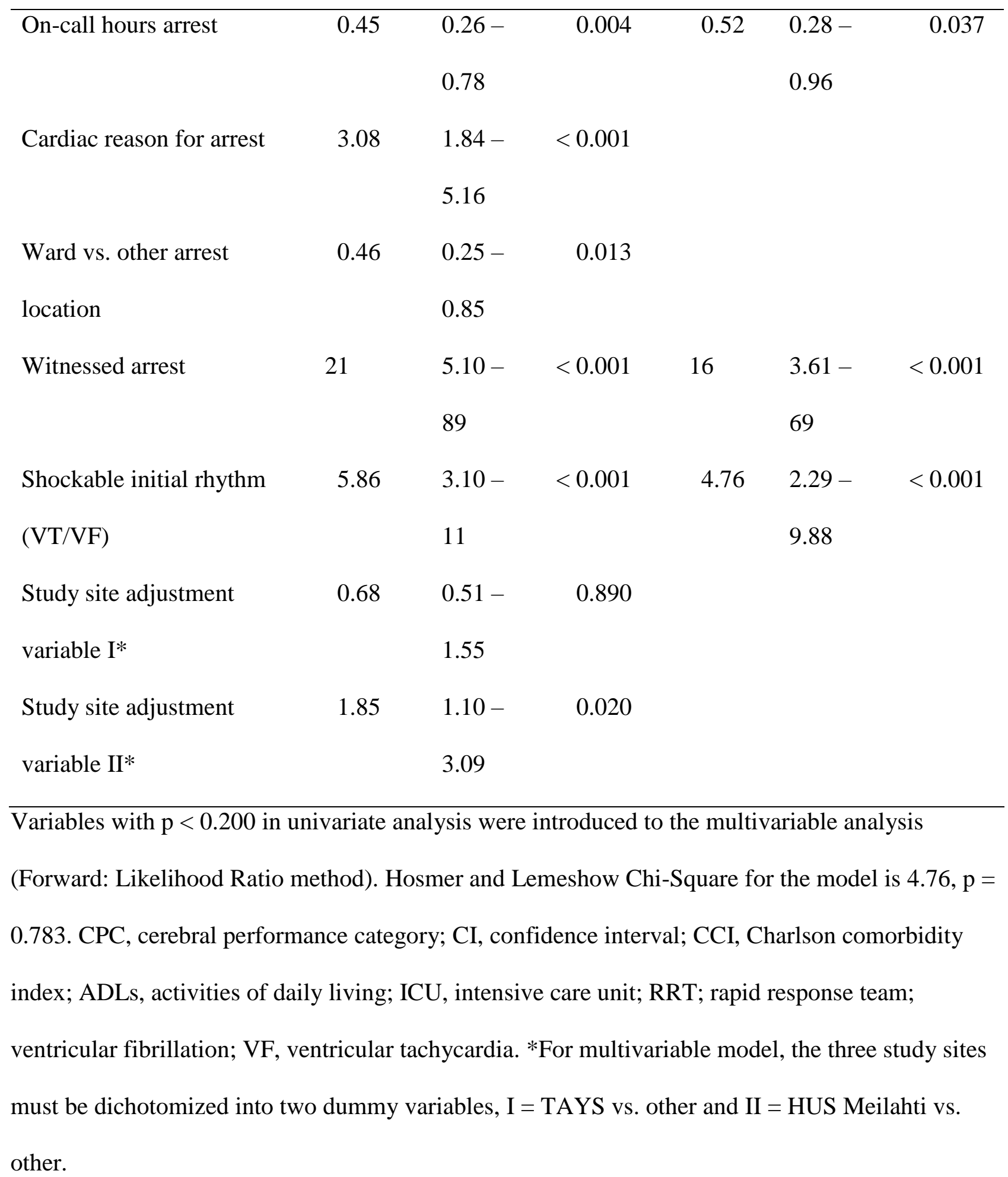


Figure 1

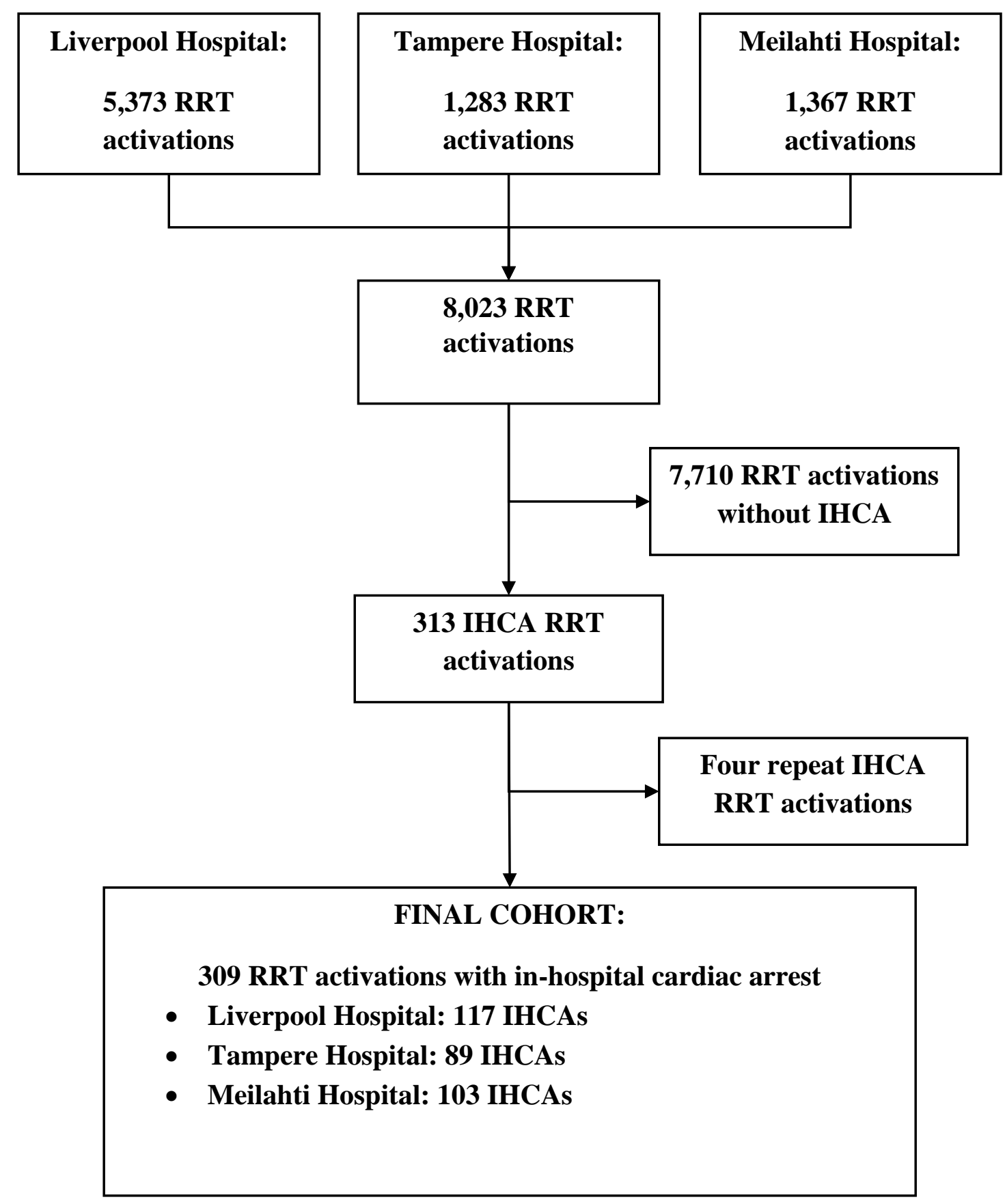


Figure 2.

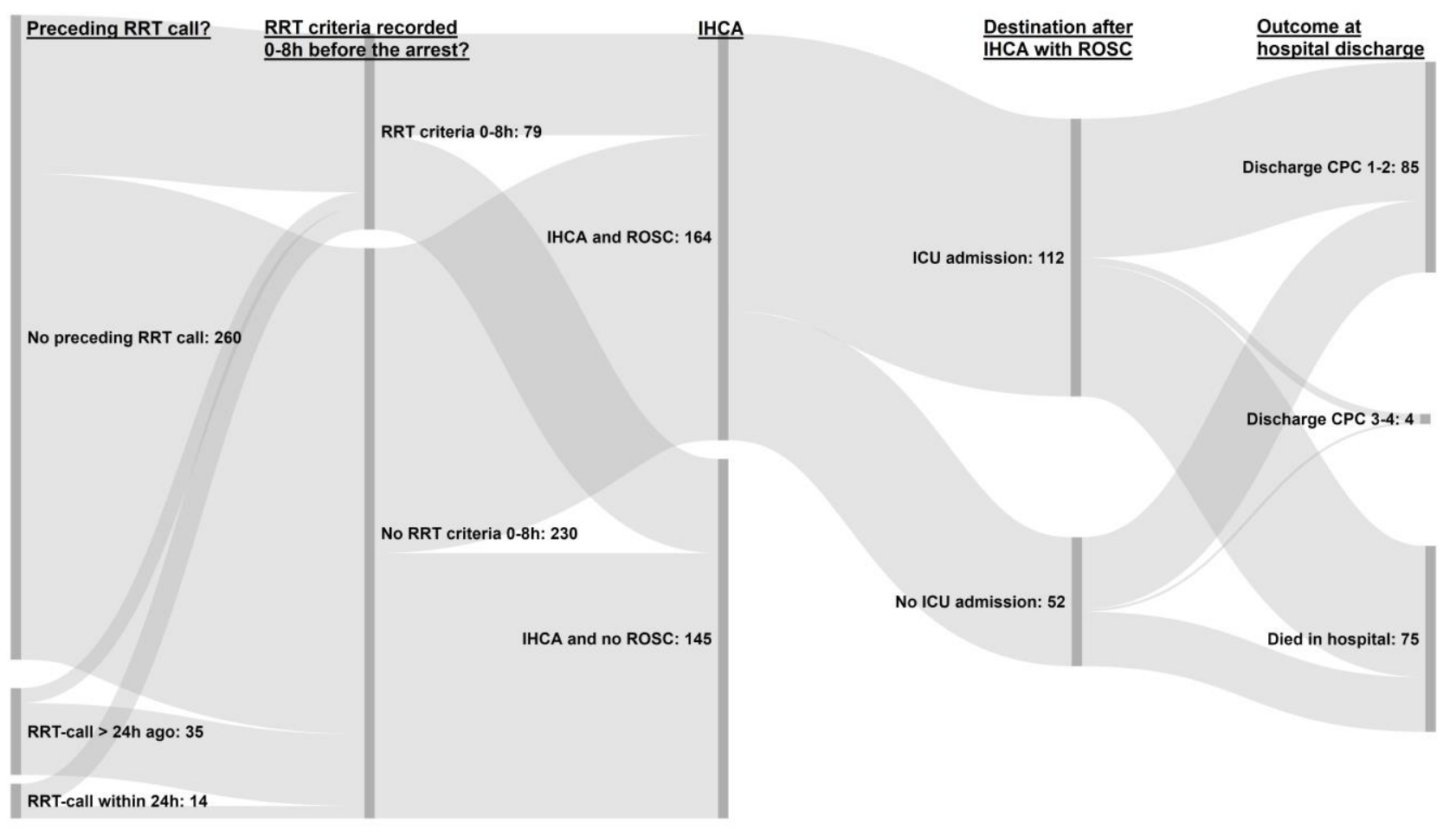


Appendix A. Hospital and rapid response system characteristics

\section{Tampere}

Helsinki Meilahti Liverpool

\begin{tabular}{|c|c|c|c|}
\hline Hospital beds & 550 & 430 & 750 \\
\hline Hospital admissions per year & 69,000 & 43,000 & 85,000 \\
\hline ICU beds & 26 & 48 & 34 \\
\hline ICU admissions per year & 2,700 & 2,700 & 3,000 \\
\hline RRT implementation year & 2009 & 2010 & 1990 \\
\hline \multirow[t]{3}{*}{ RRT team composition } & ICU Consultant/ & ICU Consultant/ & ICU Registrar \\
\hline & Registrar \& two & Registrar \& two & \& two ICU \\
\hline & ICU nurses & ICU nurses & nurses \\
\hline Adult RRT activations per 1,000 & 9.3 & 16 & 32 \\
\hline hospital admissions per year & & & \\
\hline $\begin{array}{l}\text { Adult IHCAs per 1,000 hospital } \\
\text { admissions per year }\end{array}$ & 0.64 & 1.20 & 0.69 \\
\hline \multicolumn{4}{|l|}{ RRT activation criteria } \\
\hline $\begin{array}{l}\text { Respiratory rate } \\
\text { (breaths/min) }\end{array}$ & $<5$ or $>24$ & $<5$ or $>28$ & $<5$ or $>30$ \\
\hline $\mathrm{SpO}_{2}(\%)$ & $<90$ & $<90$ & $<90$ \\
\hline Heart rate (beats/min) & $<40$ or $>140$ & $<40$ or $>140$ & $<40$ or $>140$ \\
\hline $\begin{array}{l}\text { Systolic blood pressure } \\
\text { (mmhg) }\end{array}$ & $<90$ & $<90$ & $<90$ or $>200$ \\
\hline \multirow[t]{2}{*}{ Neurology } & Fall in GCS $\geq 2$ & 'Sudden fall in & Fall in GCS $\geq$ \\
\hline & & GCS' & 3 \\
\hline
\end{tabular}




\begin{tabular}{|c|c|c|c|}
\hline Nurse worried criterion & Included & Included & Included \\
\hline \multicolumn{4}{|l|}{ Between the flags criteria } \\
\hline Respiratory rate & & & $5-10$ or $25-30$ \\
\hline \multicolumn{4}{|l|}{ (breaths/min) } \\
\hline $\mathrm{SpO}_{2}(\%)$ & & & $90-95$ \\
\hline \multirow[t]{2}{*}{ Heart rate (beats/min) } & N/A & N/A & $40-50$ or $120-$ \\
\hline & & & 140 \\
\hline Systolic blood pressure & & & $90-100$ or $180-$ \\
\hline (mmhg) & & & 200 \\
\hline Neurology & & & New confusion \\
\hline Body temperature (C) & & & $<35.5$ or $>38.5$ \\
\hline \multirow[t]{3}{*}{ Urine output } & & & Anuria, \\
\hline & & & $<200 \mathrm{ml} / 8$ \\
\hline & & & hours \\
\hline Blood glucose (mmol/l) & & & $1-4$ \\
\hline New/uncontrolled pain & & & Included \\
\hline Excessive fluid loss (drains, & & & Included \\
\hline \multicolumn{4}{|l|}{ polyuria) } \\
\hline Nurse worried criterion & & & Included \\
\hline
\end{tabular}

Data are given as numbers if not otherwise indicated. ICU, intensive care unit; RRT, rapid response team; IHCA, in-hospital cardiac arrest, GCS, Glasgow coma scale. 
Appendix B. Data from individual study sites.

\begin{tabular}{|c|c|c|c|}
\hline & \multicolumn{2}{|c|}{ Patient characteristics } & Liverpool \\
\hline Age & $75(62,82)$ & $67(58,78)$ & $73(60,82)$ \\
\hline Sex (male) & $71(63)$ & $59(61)$ & $61(71)$ \\
\hline CPC $>2$ before hospital admission & $0.0(0)$ & $0.0(0)$ & $5.1(6)$ \\
\hline \multicolumn{4}{|l|}{$\begin{array}{l}\text { Performance in ADLs before hospital } \\
\text { admission }\end{array}$} \\
\hline Lives fully independently & $74(66)$ & $85(87)$ & $62(73)$ \\
\hline Lives home with support in ADLs & $23(20)$ & $14(14)$ & $31(36)$ \\
\hline $\begin{array}{l}\text { Lives in nursing home, support in all } \\
\text { ADLs }\end{array}$ & $3.4(3)$ & $1.9(2)$ & $6.8(8)$ \\
\hline HDS & $2(1,3)$ & $2(1,4)$ & $2(1,4)$ \\
\hline Coronary artery disease & $25(22)$ & $44(45)$ & $39(46)$ \\
\hline Peripheral arterial disease & $12(11)$ & $16(16)$ & $13(15)$ \\
\hline Diabetes & $32(28)$ & $29(30)$ & $44(52)$ \\
\hline $\begin{array}{l}\text { Chronic obstructive pulmonary } \\
\text { disease }\end{array}$ & $11(10)$ & $10(10)$ & $13(15)$ \\
\hline Malignancy & $20(18)$ & $18(18)$ & $15(18)$ \\
\hline \multicolumn{4}{|l|}{ Hospital admission characteristics } \\
\hline Outpatient visiting a clinic & $4.5(4)$ & $5.8(6)$ & $4.3(5)$ \\
\hline Surgical reason for admission & $48(43)$ & $44(45)$ & $28(33)$ \\
\hline Days in hospital before the IHCA & $2(1,5)$ & $2(0,7)$ & $4(1,17)$ \\
\hline Preceding RRT call & $6.7(6)$ & $6.8(7)$ & $31(36)$ \\
\hline Within 24 hours before the IHCA & $3.4(3)$ & $1.9(2)$ & $7.7(9)$ \\
\hline Preceding ICU admission & $12(11)$ & $17(17)$ & $17(20)$ \\
\hline $\begin{array}{l}\text { Discharged within } 0-8 \text { hours before } \\
\text { IHCA }\end{array}$ & $3.4(3)$ & $0.0(0)$ & $1.7(2)$ \\
\hline PCI/PCA 0-8 hours before IHCA & $15(13)$ & $27(28)$ & $10(12)$ \\
\hline Surgery 0-8 hours before IHCA & $4.5(4)$ & $0(0.0)$ & $0.9(1)$ \\
\hline Preceding DNAR order in place & $2.2(2)$ & $2.9(3)$ & $10(12)$ \\
\hline $\begin{array}{l}\text { Objective RRT activation criteria without } \\
\text { subsequent RRT activation recorded 0-8 } \\
\text { hours before IHCA }\end{array}$ & $38(34)$ & $28(29)$ & $14(16)$ \\
\hline Respiratory criteria & $30(27)$ & $11(11)$ & $5.1(6)$ \\
\hline Circulatory criteria & $12(11)$ & $15(15)$ & $7.7(9)$ \\
\hline Neurological criteria & $10(9)$ & $4.8(5)$ & $1.7(2)$ \\
\hline \multicolumn{4}{|l|}{ IHCA event characteristics } \\
\hline \multicolumn{4}{|l|}{ RRT activation reason } \\
\hline Cardiac arrest & $81(72)$ & $97(100)$ & $84(97)$ \\
\hline Respiratory criteria & $3.4(3)$ & $1.9(2)$ & $6.9(8)$ \\
\hline Haemodynamic criteria & $4.5(4)$ & $0.0(0)$ & $4.3(5)$ \\
\hline Neurological criteria & $9.0(8)$ & $0.1(1)$ & $5.2(6)$ \\
\hline Nurse worried & $1.1(1)$ & $0.0(0)$ & $0.0(0)$ \\
\hline Other & $1.1(1)$ & $0.0(0)$ & $0.0(0)$ \\
\hline \multicolumn{4}{|l|}{ Location } \\
\hline Ward & $88(78)$ & $76(78)$ & $86(100)$ \\
\hline
\end{tabular}




\begin{tabular}{|c|c|c|c|}
\hline Clinic & $2.2(2)$ & $1.0(1)$ & $4.3(5)$ \\
\hline Angiology / Radiology & $4.5(4)$ & $21(22)$ & $7.7(9)$ \\
\hline Emergency room & $0.0(0)$ & $1.9(2)$ & $0.9(1)$ \\
\hline OR / PACU & $5.6(5)$ & $0.0(0)$ & $1.7(2)$ \\
\hline Arrest during on-call hours & $72(64)$ & $75(77)$ & $73(85)$ \\
\hline \multicolumn{4}{|l|}{ Situation at RRT arrival } \\
\hline Patient on cardiac arrest & $57(51)$ & $85(87)$ & $82(96)$ \\
\hline Patient already with ROSC & $24(21)$ & $5.8(6)$ & $5.1(6)$ \\
\hline Cardiac arrest after RRT arrival & $19(17)$ & $9.7(10)$ & $13(15)$ \\
\hline Witnessed arrest & $80(71)$ & $82(84)$ & $65(76)$ \\
\hline \multicolumn{4}{|l|}{ Reason for arrest } \\
\hline Cardiac & $40(36)$ & $45(46)$ & $33(39)$ \\
\hline Respiratory & $21(19)$ & $6.8(7)$ & $21(25)$ \\
\hline Other & $38(34)$ & $49(50)$ & $45(53)$ \\
\hline \multicolumn{4}{|l|}{ Initial rhythm } \\
\hline Ventricular fibrillation & $11(10$ & $17(17)$ & $9.4(11)$ \\
\hline Ventricular tachycardia & $3.4(3)$ & $2.9(3)$ & $6.0(7)$ \\
\hline Pulseless electrical activity & $57(51)$ & $44(45)$ & $44(51)$ \\
\hline Asystole & $27(24)$ & $23(24)$ & $36(42)$ \\
\hline Unknown & $1.1(1)$ & $14(14)$ & $5.1(6)$ \\
\hline Duration of resuscitation attempt (min) & $7(4,15)$ & $11.5(4,21)$ & $10(4,20)$ \\
\hline Defibrillation at any time during CPR & $18(16)$ & $36(37)$ & $24(28)$ \\
\hline Adrenaline at any time during CPR & $58(52)$ & $69(71)$ & $75(88)$ \\
\hline Amiodarone at any time during CPR & $3.4(4)$ & $11(11)$ & $2.6(3)$ \\
\hline \multicolumn{4}{|l|}{ Airway management } \\
\hline No airway management & $30(27)$ & $1.0(1)$ & $12(14)$ \\
\hline Bag mask ventilation & $55(49)$ & $86(89)$ & $86(101)$ \\
\hline Laryngeal mask & $5.6(5)$ & $1.0(1)$ & $33(38)$ \\
\hline Endotracheal intubation & $46(41)$ & $70(72)$ & $36(42)$ \\
\hline Tracheostomy already in place & $1.1(1)$ & $1.0(1)$ & $0.9(1)$ \\
\hline Lucas ${ }^{\circledR}$ used & $1.1(1)$ & $16(16)$ & $2.6(3)$ \\
\hline ECPR initiated & $0.0(0)$ & $2.9(3)$ & $0.9(1)$ \\
\hline Thoracostomy/ re-sternotomy conducted & $2.2(2)$ & $3.9(4)$ & $0.0(0)$ \\
\hline \multicolumn{4}{|l|}{ Patient outcomes } \\
\hline ROSC & $46(41)$ & $65(63)$ & $50(58)$ \\
\hline \multicolumn{4}{|l|}{ Post ROSC location } \\
\hline Stays on arrest location & $4.9(2)$ & $6.2(4)$ & $1.7(1)$ \\
\hline OR or PACU only & $20(8)$ & $9.2(6)$ & $17(10)$ \\
\hline Higher monitoring unit & $15(6)$ & $20(13)$ & $3.4(2)$ \\
\hline ICU & $61(25)$ & $65(42)$ & $78(45)$ \\
\hline ICU length of stay & $2(1,3)$ & $3(1,9)$ & $4(1,8)$ \\
\hline ICU survival & $60(15)$ & $73(32)$ & $42(19)$ \\
\hline 24-hour survival & $38(34)$ & $47(48)$ & $37(43)$ \\
\hline Survival to hospital discharge & $27(24)$ & $39(40)$ & $21(25)$ \\
\hline $\begin{array}{l}\text { Survival to hospital discharge with } \\
\text { CPC } 1-2\end{array}$ & $26(23)$ & $36(37)$ & $21(25)$ \\
\hline
\end{tabular}

Data are presented as percentages (counts) and continuous variables as medians $\left(\mathrm{Q}_{1}, \mathrm{Q}_{3}\right)$. CPC, cerebral performance category; ADLs, activities in daily living; CCI, Charlson comorbidity index; IHCA, in-hospital cardiac arrest; RRT, rapid response team; ICU, intensive care unit; PCA, 
percutaneous coronary angiography; PCA, percutaneous coronary intervention; DNAR, do not attempt resuscitation order, OR, operating room; PACU, post anaesthesia care unit; ROSC, return of spontaneous circulation; CPR, cardiopulmonary resuscitation; Lucas ${ }^{\circledR}$, Lund University Cardiopulmonary Assist System; ECPR, extracorporeal cardiopulmonary resuscitation. 